higher proportion of diarrhoeal samples tested positive to Campylobacter (12\%) compared to the non-diarrhoeal control group (5\%). Unlike the diarrhoeal samples, no control samples yielded positivity to Vibrio cholerae, Cyclospora cayetanensis, Astrovirus, Rotavirus A or Sapovirus

Conclusions Detection of high levels of polymicrobial enteric infections are prevalent in Central Indian symptomatic and asymptomatic populations. E. coli pathotypes predominate in both urban and rural settings. Further studies are required to understand the clinical significance of these mixed infections, as well as how best to manage them.

\section{PTH-92 BRAIN-DERIVED NEUROTROPHIC FACTOR (BDNF) AND EPITHELIAL TO MESENCHYMAL TRANSITION (EMT), CLINICAL PERSPECTIVE IN COLORECTAL CANCERS}

1,2,3 Han Gao*, 1,2,3 Zhe Zhao, 2,3 Johannes Benedikt, 1,3 Wen G Jiang, 1,3 Tracey A Martin. ${ }^{1}$ School of Medicine, Cardiff University, Cardiff, UK; ${ }^{2}$ School of Enginnering, Cardiff University, Cardiff, UK; ${ }^{3}$ School of Medicine and School of Engineering, Cardiff University, Cardiff, UK

\subsection{6/gutjnl-2021-BSG.295}

Introduction Brain-Derived Neurotrophic Factor (BDNF), via its cognitive receptors Neurotrophic Receptor Tyrosine Kinases (NRTK/TrKs), is a neural survival factor and has been implicated in the development and progression of neurological cancer and selective non-neurological solid cancers. On cancer cells, BDNF has been reported to elicit biological influence on functions such as apoptosis, cellular motility and invasion, some of the hallmarks seen during Epithelial to Mesenchymal Transition (EMT). The present study examined the clinical and prognostic value of BDNF in connection with EMT markers, including Twist, Snail-1 (SNAI1), Slug (SNAI2), E(epithelial)-cadherin and $\mathrm{N}$ (neural)-cadherin, which were reported to have pivotal role in assessing disease progression and the clinical outcome of patients with certain solid tumours.

Methods A cohort of colorectal cancers were collected and analysed for the expression gene transcripts of Brain-Derived Neurotrophic Factor, together with a group of key EMTs biomarkers Twist, Snail-1, Slug, E-cadherin and N-cadherin. The clinical and prognostic values of the markers were collectively analysed in the connection with clinical and pathological factors and most importantly the clinical outcome of the patients.

Results The levels of BDNF transcript are significantly correlated with the Twist, Snail-1 and Slug $(p<0.001$, for all three molecules), to a lesser degree with E-cadherin $(p<0.01)$ and marginally with $\mathrm{N}$-cadherin. Whilst these markers had significant predictive effects on the clinical outcome of the patients, the combined analyses of BDNF and EMT markers together have offered a more significant power in predicting the overall survival of the patients. Patients with highly aberrant expression of BDNF/EMT markers showed a markedly shorter survival $(40.7 \%$ vs $75 \%$ vs $100 \%$ survival rate over the 6 year follow-up period, respectively for those with high, moderate and low aberrant expression) (HR 2.7, p <0.0001). Similar correlation was seen between BDNF/EMT markers with the disease free survival $(44.4 \%, 70 \%$ and $96.7 \%$ respectively, HR 2.07, $p=0.001)$. These factors are in line with other clinical and pathological factors, including tumour staging $(p=0.027)$ and Dukes staging $(p=0.017)$, in the prediction of patient's survival.
Conclusions BDNF and the biochemical markers of EMT are aberrantly expressed in clinical colorectal cancer. The degree of aberration has a clear and significant clinical value in predicting the outcome of the patients.

Martin TA, Goyal A, Watkins G, et al. Ann. Surg. Oncol., 2005, 12: 488-496

\section{PTH-93 AF6 (AFADIN/MLLT4) AND TIGHT JUNCTIONAL REGULATING SIPA1, EXPRESSION AND CLINICAL/ PROGNOSTIC VALUE IN COLORECTAL CANCER}

1,2Han Gao*, ' Chang Liu, ${ }^{2}$ Johannes Benedikt, 'Wen G Jiang, ${ }^{1}$ Tracey A Martin. 'School of Medicine, Cardiff University, Cardiff, UK; ${ }^{2}$ School of Enginnering, Cardiff University, Cardiff, UK

\subsection{6/gutjnl-2021-BSG.296}

Introduction AF6 (Afadin, also known as MLLT4) is a junctional protein that belongs to the adhesion system of regulators of cell junctional adhesion. AF6 has been indicated in embryogenesis and development of certain types of haematological malignancies, and to a limited degree in solid tumours. In colorectal cancer, AF6 has been shown to regulate Cystic Fibrosis Transmembrane Conductance Regulator (CFTR). Recently, it was revealed that SIPA1 (Signal-Induced Proliferation-Associated-1), a kinase regulating cell cycle and mitogenesis, has recently discovered to regulate tight junctions. The present study explored the expression and connection between AF6 and SIPA1 in human colorectal cancers.

Methods The expression levels of AF6 and SIPA1 in human colorectal tumour and normal tissues were quantitatively determined by way of gene transcript analyses. The expression was analysed against the clinical and pathological parameters, together with clinical outcome of the patients including recurrence, metastasis and colorectal related death. Statistical methods were Mann Whitney U test for comparisons, logistic regression and Kaplan-Meier's methods for survival analyses.

Results Both AF6 and SIPA1 had an aberrant pattern of expression in colorectal tumours compared with normal colon control tissues. Our dataset surprisingly displayed a highly significant correlation between AF6 and SIPA1 $(\mathrm{r}=0.904, \mathrm{p}<0.00001)$. AF6 and SIPA1 had significant value in predicting overall survival (Hazard Ratio (HR) 3.7, $\mathrm{p}=0.05$, for AF6 and HR 4.8, p=0.01) and relapse free survival $(\mathrm{p}=0.044$ and $\mathrm{p}=0.016$ for AF6 and SIPA1 respectively). When the expression pattern of AF6 and SIPA1 were integrated for analyses, they further enhanced the prediction power for both overall survival (mean survival 38.5 months, 111.6 months and 159.6 months respectively for the differential expression pattern, $\mathrm{p}=0.002$ ) and relapse free survival (mean survival 32.3, 108.4 and 153.3 months respectively, $\mathrm{p}=0.005)$. AF6/SIPA1 expression pattern is an independent prognostic factor for overall survival $(p=0.008)$, in contrast to staging, differentiation and nodal status which showed either independency in the current cohort. AF6/SIPA1 is a weak factor for disease free survival $(p=0.06)$, in contrast to tumour staging and nodal status which had independent predictive value $(\mathrm{p}<0.05)$.

Conclusion AF6, together with SIPA1, are aberrantly expression in clinical colorectal cancer and have significant values in predicting the progression and the outcome of the patients. The molecules are potential targets in colorectal cancer. 